colonoscopic technique from Taiwan which facilitates easier placement of the snare thereby achieving en-bloc resection.

Methods A 67 year old lady presented to our institute with an altered bowel habit and was found to have a flat polyp in the sigmoid colon on colonoscopy.

Results Standard injection as per conventional endoscopic mucosal resection was initially performed. Then a suitably sized snare was selected and the snare tip was used to make a single incision with cut current lateral to the polyp. The snare tip was then anchored at the site of the incision and then the snare was slowly opened and simultaneously positioned around the polyp. Once the snare was adequately placed the polyp was resected. Histology revealed a tubulovillous adenoma with low grade dysplasia which was excised completely.

Conclusions This technique provides an easy and safe way to resect en-bloc flat, large and challenging colonic polyps.

\section{PTH-079 FULLY COVERED METAL STENT INSERTION FOR THE TREATMENT OF REFRACTORY POST ENDOSCOPIC SPHINCTEROTOMY BLEEDING}

Yasmin Kassir*, Alvin Ochieng, Phillip Berry, Terence Wong. Guy's and St Thomas' NHS Foundation Trust, London, UK

\subsection{6/gutjnl-2018-BSGAbstracts. 102}

Introduction Bleeding is a common complication of endoscopic sphincterotomy (ES), occurring in 4\% of cases. Fully covered metal stents (FCMS) are mainly indicated in the treatment of biliary strictures, bile ducts leaks and biliary stones. Recent studies have shown the value of fully covered metal stent placement in the management of post ES haemorrhage after failure of primary endoscopic interventions.

Treatment options have previously been limited to arterial embolisation or surgery in cases where conventional endoscopic therapy has failed. FCMS placement provides a less invasive means of achieving haemostasis through mechanical tamponade and may be a suitable option in patients whose bleeding has not been controlled with first line endoscopic management.

Methods We report a case of post ES bleeding refractory to conventional therapy, where haemostasis was achieved through placement of a temporary FCMS. A 27 year old man underwent therapeutic ERCP for choledocholithiasis in which precut sphincterotomy (with needle knife) resulted in bleeding. Initial management with local injections of adrenaline, endoclips and heater probe therapy were unsuccessful in achieving prolonged haemostasis and the patient became haemodynamically unstable, with melaena and $\mathrm{Hb}$ drop from $103 \mathrm{~g} / \mathrm{L}$ to $56 \mathrm{~g} / \mathrm{L}$. The patient underwent a repeat ERCP in which a fully covered $(10 \mathrm{~mm} / 6 \mathrm{~cm})$ metal stent (Wallflex, Boston) was inserted across the ampulla to tamponade the site of bleeding. The stent remained insitu and was removed 6 weeks post initial insertion, with no residual bleeding. Of note, the patient developed acute cholecystitis 48 hours post stent insertion, requiring urgent cholecystectomy. There were no post-operative complications.

Results Our case demonstrates the successful management of post ES bleeding with the use of FCMS placement, avoiding the need for arterial embolisation or surgery. Despite achieving haemostasis, our patient developed acute cholecystitis following stent placement, requiring urgent cholecystectomy. This has been reported in up to $10 \%$ of patients with FCMS for all indications. The patient remained well post operatively and stent was removed with no residual bleeding.

Conclusion Our case supports the proposed use of FCMS placement as second line management in post ES bleeding refractory to conventional endoscopic therapy. In applying this technique we avoided the use of arterial embolisation and its associated risks and complications, of particular importance in a young patient such as ours. There is a risk of cystic duct outflow obstruction in the application of covered metal stents, as our case highlights, and it is important to recognise this when considering this treatment modali.

\section{PTH-080 A MULTIMODALITY ENDOSCOPIC APPROACH FOR MANAGEMENT OF BURIED BUMPER SYNDROME}

Nikolaos Lazaridis*, Alberto Murino, Andrea Telese, Nikolaos Koukias, Edward J Despott. Royal Free Unit for Endoscopy, The Royal Free Hospital and University College London (UCL) Institute for Liver and Digestive Health, Hampstead, London, UK

\subsection{6/gutjnl-2018-BSGAbstracts. 103}

Introduction Buried bumper syndrome (BBS) is a rare, longterm complication of percutaneous endoscopic gastrostomy (PEG) placement, occurring in $2 \%-6 \%$ of the cases. BBS is thought to occur due to prolonged compression of the tissue between the external and internal fixators, leading to 'burying' of the PEG bumper into the gastric wall. Consequences of BBS include tube obstruction and more rarely bleeding, abscess formation, and perforation. Several endoscopic techniques are described for the management of BBS and these may be complimentary when used in combination.

Methods A 32-year-old woman with diabetes, chronic kidney disease, a history of hypoglycaemic brain injury and gastroparesis, requiring a venting PEG, presented with abdominal pain. PEG tube obstruction led to the suspicion of BBS and abdominal computerised tomography confirmed this.

Results At upper gastrointestinal endoscopy under general anaesthesia, the internal bumper was found to be completely buried by granulation and fibrotic tissue. A $2.5 \mathrm{~mm}$ FlushKnife (Fujifilm, Saitama, Japan) was initially used to partially dissect the overgrown gastric tissue in order to achieve insertion of a biopsy forceps down the external aspect of the PEG tube and through the dissected orifice. This manoeuvre opened a track in the overgrown tissue for insertion of a sphincterotome mounted on a JagWire (Boston Sci., MA, USA) through the external PEG tube. The sphincterotome was then flexed completely and several radial incisions on the overgrown tissue were performed using external traction on the sphincterotome. Finally, a $6 \mathrm{~mm}$ endoscopic balloon dilator was passed through the scope and pulled into the PEG tube by the biopsy forceps inserted through the external end of the tube. The balloon was then fully inflated within the PEG tube and traction was applied to the balloon and endoscope for release of the buried bumper and PEG tube remnant from the dissected overgrown tissue into the stomach. The dissected orifice was then closed using endoscopic clips. The procedure was performed under antibiotic prophylaxis.

Conclusion To the best of our knowledge, this is the first use of a complimentary, multimodality endoscopic approach for the effective, minimally invasive, safe management of BBS. 